\title{
Effect of Cocaine Use on Bone Marrow-mediated Erythropoiesis
}

\author{
Jim Edward Weber, DO, Gregory Luke Larkin, MD, MS, MSPH, \\ Christopher T. Boe, MD, Andrew Fras, MSHE, Amit S. Kalaria, MD, \\ Ronald F. Maio, DO, MS, Benjamin Luchessi, MD, PhD, \\ Lars Ensign, MD, Brian Sweeney, DO, Judd E. Hollander, MD
}

\begin{abstract}
Objectives: Cocaine-mediated erythrocytosis is one of several effects that cocaine may have on hematologic indices; however, the precise mechanism by which cocaine induces peripheral erythrocytosis is not fully understood. The objective of this study was to examine the contribution of the bone marrow to cocaine-mediated erythrocytosis. Methods: Differences in mean hemoglobin concentration, hematocrit, and reticulocyte counts were measured in consecutive cocaine-exposed and cocaine-unexposed patients who presented to the emergency department (ED) with chest pain. Acute cocaine exposure ( $<3$ hours) was confirmed by history and toxicologic analysis of the urine on all patients. Means were compared using independent-samples t-test and covariates were examined using multiple regression. Results: Seventy-nine patients met enrollment criteria. Hemoglobin and hematocrit levels were significantly elevated in the cocaine-using subjects $(13.5 / 39.8)$ compared with controls $(12.6 / 37.7 ; \mathrm{p}<0.02)$. However, no corresponding elevation in
\end{abstract}

reticulocyte count $(p=0.2)$ was observed. Multivariate logistic regression revealed that male chest pain patients were significantly more likely to be exposed to cocaine (OR $5.15[95 \% \mathrm{CI}=1.77$ to 15.3$])$ than females $(\mathrm{p}=0.001)$, and all relative increases in hemoglobin concentration in the cocaineexposed group were attributable to gender. Cocaine exposure was not significantly associated with reticulocyte count. Multivariate linear regression revealed that of demographic, medical, and substance use covariates, only a history of diabetes mellitus was significantly associated with an elevated reticulocyte count $(p=0.009)$. Conclusions: Acute cocaine exposure is not associated with erythrocytosis in younger ED patients with chest pain. The lack of an elevated reticulocyte count suggests that bone marrow does not contribute to any transient erythrocytosis that may occur. Key words: cocaine; chest pain; erythropoiesis; reticulocytosis. ACADEMIC EMERGENCY MEDICINE 2003; 10:705708.
Chest pain is the most frequent cardiac consequence of cocaine abuse reported in the emergency department (ED), ${ }^{1}$ with acute myocardial infarction occurring in as many as $6 \%$ of such patients who present to an ED for care. ${ }^{2,3}$ Numerous factors have been implicated in the pathophysiology of cocaineassociated myocardial ischemia, including coronary

From the Departments of Emergency Medicine, University of Michigan Medical School, Ann Arbor, MI, and Hurley Medical Center, Flint, MI (JEW, AF, RFM, LE); Department of Emergency Medicine, University of Texas Southwestern Medical School, Dallas, TX (GLL); Department of Emergency Medicine, Saginaw Cooperative Hospitals/Michigan State University College of Human Medicine, East Lansing, MI (CTB); Department of Emergency Medicine, Northwestern University, Chicago, IL (ASK); Department of Pharmacology, University of Michigan Medical School, Ann Arbor, MI (BL); Pontiac Osteopathic Hospital, Pontiac, MI (BS); and Department of Emergency Medicine, University of Pennsylvania Medical School, Philadelphia, PA (JEH).

Received October 25, 2002; revisions received December 19 and 23, 2002; accepted January 14, 2003.

Presented at the SAEM annual meeting, St. Louis, MO, May 2002. Address for correspondence and reprints: Jim Edward Weber, DO, Director of Research, Department of Emergency Medicine, One Hurley Plaza, Flint, MI 48503. Fax: 810-760-0853; e-mail: jwmd@ umich.edu. vasoconstriction, ${ }^{4}$ platelet aggregation, ${ }^{5-8}$ and premature atherosclerosis. ${ }^{6,9-11}$ In-situ thrombus formation may occur as a result of altered plasma constituents that influence thrombogenicity. ${ }^{9,12-14}$ Recently, Seigel and colleagues reported that cocaine use acutely increases hemoglobin and hematocrit levels 4-6\% above baseline, ${ }^{15}$ an increase quantitatively similar to infusion of 2 units of packed red blood cells (RBCs). ${ }^{16}$ The relative contributions of these pathophysiologic mechanisms are only partly understood. Cocaine induces splenic constriction in humans, which may contribute temporally to concordant hematologic parameter changes. ${ }^{17}$ However, the degree of cocaine-induced splenic constriction does not appear to be able to fully account for the subsequent increases in hemoglobin and hematocrit levels, suggesting that other sequestered, concentrated, RBC pools of multiple origins are liberated by cocaine administration. ${ }^{17}$ A more complete picture may be gleaned by considering the relative contribution of the bone marrow in cocaine-induced erythrocytosis, although this mechanism has not been previously investigated. Our hypothesis was that the bone marrow contribution to cocaine-induced erythrocytosis would be negligible. 


\section{METHODS}

Study Design. This was a prospective observational study. The protocol was approved by the Hurley Medical Center Institutional Review Board prior to study commencement, and informed consent was provided by all subjects.

Study Setting and Population. This study was conducted at an urban university-affiliated trauma center with an annual volume of 80,000 patients. Data were collected from consecutive patients (cocaineusing and non-cocaine using) aged 18-50 years who presented to the ED cardiac diagnostic unit (CDU) with a chief complaint of chest pain and received a diagnostic workup for ischemia. Cocaine-using patients required a history of cocaine use within three hours prior to presentation. Subjects who tested positive for cocaine but denied use were further questioned regarding cocaine use. Only those who ultimately admitted to use within three hours of ED presentation were included in the analysis.

Patients were excluded if they were pregnant, incarcerated, residents of a mental institution, or otherwise unable to provide informed consent. Also excluded were patients at high risk for cardiovascular deterioration, defined by triage to the coronary care unit (CCU), cardiac telemetry, or the cardiac catheterization laboratory. In addition, patients with sickle cell disease, malignancy, history of marrow transplantation, immunosuppression, gastrointestinal bleeding, chronic obstructive pulmonary disease, renal failure, or myeloproliferative disorders (e.g., polycythemia vera) were also excluded.

Study Protocol. A detailed discussion of the CDU protocol was previously published by Gibler and colleagues. ${ }^{18}$ In accordance with our institutional standard of care, urine was routinely analyzed toxicologically on all ED patients $<50$ years of age with a chief complaint of chest pain presumed to be of ischemic origin, and all patients were evaluated in the CDU.

Measurements. Patient demographics (age, gender, and ethnic group), past history including cardiac risk factors (hypertension, hypercholesterolemia, tobacco use, type I and or type II diabetes mellitus, and family history of premature atherosclerotic heart disease), and the route of cocaine use (nasal, intravenous, or inhalation/crack) were recorded.

Hematologic parameters were determined using a blood cell analyzer (Miles/Bayer H2 System; Smith Kline Beecham Clinical Laboratories, Waltham, MA). Complete reticulocyte count $(\% \mathrm{CRC})$ was defined as the percentage of total reticulocytes. Absolute reticulocyte count (ARC) was defined as the number of reticulocytes present in $1 \mathrm{~mm}^{3}$ of blood, as was calculated as (reticulocyte \%)/100 $\times$ erythrocyte count. The main outcome was the change in CRC between cocaine-using and non-using chest pain patients.

Data Analysis. Reticulocytes comprise $0.5-2.0 \%$ of the total erythrocyte count in adults. We sought to detect a difference that was at the lower limit of normal, or smaller, in order to refute the null hypothesis. A sample of 34 patients in each group yielded a $90 \%$ power of detecting a difference in \%CRC of 0.35 between groups, which is even less than a clinically meaningful difference of 0.5 , the lower limit of normal. Continuous variables are reported as means \pm standard deviation $( \pm \mathrm{SD})$. Categorical variables are reported as the percentage frequency of occurrence. Baseline differences between cohorts were controlled for using stratification and multivariate analysis of variance, where appropriate. After sufficient numbers of smokers were enrolled, we extended enrollment time in order to capture additional (smoking and nonsmoking) patients with cocaine exposure in order to ensure adequate control for tobacco exposure. Mean hemoglobin $(\mathrm{g} / \mathrm{dL})$, hematocrit $(\%)$, and reticulocyte counts were compared using two-tailed independentsamples t-test. Candidate predictors of hemoglobin and \%CRC were tested using univariate and multivariate linear regression; multiple comparisons were controlled for using the method of conferring. Differences of proportion were analyzed using chi-square and Fisher's exact test, where appropriate. Predictors of cocaine exposure were examined using multivariate logistic regression; logistic regressions were tested for fit using Hosmer-Lemeshow bounds. Data were analyzed using SAS statistical software (Version 8.0, SAS Institute, Cary, NC).

\section{RESULTS}

Baseline demographic comparisons are presented in Table 1 . Seventy-nine patients were enrolled. Thirtyfour patients tested positive for cocaine, and 45 patients were cocaine-negative. No differences were noted between groups when comparing the two groups for age, ethnicity, and cardiac risk factors. Men were significantly more likely to be exposed to cocaine than women $(64 \%$ vs $26 \%$; odds ratio [OR] $5.15 ; 95 \%$ confidence interval $[95 \% \mathrm{CI}]=1.77$ to 15.3 ; Yates-corrected chi-square 10.22; $\mathrm{p}=0.001$ ), and had significantly higher hemoglobin (13.7 vs $12.3 \mathrm{~g} / \mathrm{dL}$; two-tailed independent-samples t-test; $\mathrm{p}<0.02$ ) (Table 2.) Crack was the most frequent route of use among cocaine users (88.2\%). Univariate linear regression analysis revealed that cocaine exposure was significantly associated with an increased hemoglobin level (coefficient $\beta$ : 0.88 [95\% CI $=0.4$ to 1.4$]$; $\mathrm{p}=$ 0.002). A stepwise multivariate linear regression model, controlling for age, race, antecedent illness, 
TABLE 1. Demographics of Cocaine-positive (+) vs. Cocaine-negative ( $(-)$ Patients

\begin{tabular}{|c|c|c|c|}
\hline & $\begin{array}{c}\text { Cocaine }(+) \\
(n=34)\end{array}$ & $\begin{array}{c}\text { Cocaine }(-) \\
(n=45)\end{array}$ & $\mathrm{p}$-value* \\
\hline $\begin{array}{l}\text { Age (years- } \\
\text { mean } \pm S D)\end{array}$ & $38.2 \pm 8.9$ & $40.8 \pm 8.5$ & 0.19 \\
\hline $\begin{array}{l}\text { Gender } \\
\text { (\% male) }\end{array}$ & $67.6 \%(23)$ & $29.1 \%(13)$ & 0.001 \\
\hline $\begin{array}{l}\text { Race } \\
\text { (\% African }\end{array}$ & & & \\
\hline American) & $79.4 \%(27)$ & $73.3 \%(33)$ & 0.56 \\
\hline Tobacco (\%) & $85.2 \%(29)$ & $69.0 \%(31)$ & 0.10 \\
\hline $\begin{array}{l}\text { History of } \\
\text { diabetes (\%) }\end{array}$ & $3.2 \%(1)$ & $11.0 \%(5)$ & 0.26 \\
\hline $\begin{array}{l}\text { History of } \\
\text { hypertension } \\
\text { (\%) }\end{array}$ & $17.6 \%(6)$ & $26.6 \%(12)$ & 0.38 \\
\hline $\begin{array}{l}\text { History of } \\
\text { coronary } \\
\text { artery disease }\end{array}$ & & & \\
\hline (\%) & $3.2 \%(1)$ & $13.3 \%(6)$ & 0.09 \\
\hline Family history & $26.7 \%(9)$ & $40.0 \%(18)$ & 0.24 \\
\hline $\begin{array}{l}\text { Hypercholes- } \\
\text { terolemia }\end{array}$ & $9.0 \%(3)$ & $17.7 \%(8)$ & 0.260 \\
\hline
\end{tabular}

*Two-tailed independent-samples t-test, Yates'-corrected chisquare, or Fisher's exact test, where appropriate.

tobacco use, and interactions between gender and substance use, reveals male gender to be the strongest independent predictor of an increased hemoglobin concentration, explaining nearly a third of the variance in hemoglobin levels $\left(\mathrm{R}^{2}=0.32 ; \beta=1.31\right.$; $[95 \% \mathrm{CI}=0.79$ to 1.8$] ; \mathrm{t}=5.06)$. However, when cocaine use is added to the equation, $\mathrm{R}^{2}$ only increases 0.02 to 0.34 , illustrating that gender contributes far more to hemoglobin concentration that cocaine exposure, per se.

Cocaine exposure had a nonsignificant association with \%CRC and all covariates were insignificant with the exception of diabetes mellitus $(\mathrm{p}=0.009)$. The mean \%CRC and reticulocyte count for the cohort as a whole were 1.35 and $0.063 \times 10^{-6}$, but in diabetic patients they were significantly higher at 1.96 and 0.09 $\times 10^{-6}$, respectively $(\mathrm{p}<0.01)$.

\section{TABLE 2. Hematologic Parameters for Cocaine-} positive $(+)$ vs. Cocaine-negative $(-)$ Patients*

\begin{tabular}{lccc}
\hline & Cocaine $(+)$ & Cocaine $(-)$ & p-value \\
\hline $\begin{array}{l}\text { Hemoglobin }(\mathrm{g} / \mathrm{dL}) \\
\quad 12.0-16.0\end{array}$ & $13.5 \pm 1.1$ & $12.6 \pm 1.2$ & $0.002 \dagger$ \\
$\begin{array}{l}\text { Hematocrit (\%) } \\
\quad 37.0-47.0\end{array}$ & $39.8 \pm 3.3$ & $37.7 \pm 3.9$ & $0.014 \dagger$ \\
$\begin{array}{c}\text { Complete reticulocyte } \\
\text { count (\%) 0.5-2.0 }\end{array}$ & $1.26 \pm 0.4$ & $1.41 \pm 0.5$ & 0.20 \\
$\begin{array}{c}\text { Reticulocyte } \times 10^{-6} \\
(\mathrm{~m} / \mathrm{UL})\end{array}$ & $0.07 \pm 0.05$ & $0.06 \pm 0.02$ & 0.35 \\
\hline
\end{tabular}

*Group means \pm standard deviation.

tSignificant two-tailed independent-samples t-test for equality.

\section{DISCUSSION}

After controlling for demographic variables and cardiac risk factors, cocaine exposure was not predictive of an elevated hemoglobin or hematocrit. This is in contrast to previous, elegantly conducted experimental trials that demonstrated a significant and immediate increase in hemoglobin and hematocrit above baseline with cocaine exposure. ${ }^{15,17}$

Seigel and colleagues reported a $4-6 \%$ increase in hemoglobin and hematocrit over baseline in an experimental model with subjects receiving either intravenous or intranasal cocaine. ${ }^{15}$ Correspondingly, Kaufman and colleagues demonstrated that cocaine induces a transient constriction of the spleen that is temporally concordant with both plasma cocaine levels and hematologic parameter changes. ${ }^{17}$ Our inability to demonstrate cocaine-induced erythocytosis in the present study is likely explained by methodologic differences in study design. Both previously mentioned experimental trials measured changes in hematologic parameters at baseline in healthy volunteers, and again at various time intervals, with elevations in hemoglobin and hematocrit noted within 10 minutes of cocaine administration, decreasing toward baseline levels within 30 minutes. We evaluated a real-world clinical cohort of chest pain patients admitting to cocaine use within three hours of last use. It is possible that chestpain itself is associated with catecholamine-induced hemoconcentration that may have masked any impact of cocaine alone on measured hematologic indices. It is unlikely that meaningful increases in hemoglobin and hematocrit levels with cocaine use are quantifiable given the limitations of a clinical practice environment. Our data, however, appear to support that any erythrocytosis that occurs in response to cocaine exposure is transient and likely to be clinically insignificant in patients with chest pain. The significantly increased reticulocyte counts in the small group of diabetic patients within this cohort were unexpected; however, recent evidence suggests that autoimmune diseases such as type I diabetes are intimately linked to the bone marrow's hematopoietic system. ${ }^{19}$ Because deficient, autoreactive T cells are clonally stimulated to attack pancreatic islet cells in diabetics, defective hematopoietic regulatory mechanisms may be triggered at the marrow level. ${ }^{20}$ Future work with diabetic patients should explore the nature of this marrow stimulation and the role of erythropoietin, insulin, and related endocrinologic factors on hematologic indices.

\section{LIMITATIONS}

Several study limitations are noteworthy. This study was nonrandomized and not designed to ascertain the clinical significance of small increases in hemoglobin attributable to cocaine use. This may be clinically 
relevant since other studies have noted that other hematologic parameters, including vonWillebrand factor, also increase with cocaine exposure, without a compensatory change in endogenous fibrinolysis. ${ }^{15,21}$ In this observational cohort of chest pain patients, we were unable to control for the frequency, dose, route, or purity of cocaine used. In addition, hematologic changes detected from venous blood samples may not exactly reflect arterial blood values, so the exact effect of cocaine on arterial blood hematologic parameters cannot be ascertained by present data. The generalizability of our results is limited to low-intermediate-risk ED patients presenting with a complaint of chest pain and cocaine use. The study was likely underpowered to demonstrate significant demographic differences between cocaine-using and non-using chest pain patients. Previous studies have revealed that cocaine-using chest pain patients are more likely to be younger, male, and tobacco smokers, when compared with traditional chest pain patients. ${ }^{2}$ Consecutive enrollment of a sample that was largely African American, male smokers also makes stratification of demographic subgroups difficult, but this is precisely the group that is most at risk in clinical practice.

Although the study design did not enable us to capture the change in hemoglobin and hematocrit that can occur with cocaine use, one would not expect the reticulocyte count to be affected by this lack of sensitivity, because the lifespan of reticulocytes in peripheral blood is $24-48$ hours. ${ }^{22}$ Thus, any corresponding increase in reticulocyte count as a result of cocaine exposure would be expected to remain elevated within three hours of last use. However, we could detect no difference in \%CRC or ARC by cocaine exposure. Based on these data, the bone marrow does not appear to account for an appreciable or lasting degree of cocaine-induced erythrocytosis beyond that due to splenic constriction alone.

\section{CONCLUSIONS}

We were unable to document any erythrocytosis between cocaine-using and non-using groups in our study. Any transient erythrocytosis that may occur with cocaine exposure does not appear to be mediated by bone marrow, since reticulocytosis did not occur. Future research should pursue other erythrocyte donor pools to investigate mechanisms for erythropoesis in cocaine users other than splenic constriction.

\section{References}

1. Minor RL Jr, Scott BD, Brown DD, Winniford MD. Cocaineinduced myocardial infarction in patients with normal coronary arteries. Ann Intern Med. 1991; 115:797-806.
2. Hollander JE, Hoffman RS, Gennis P, et al. Prospective multicenter evaluation of cocaine associated chest pain. Acad Emerg Med. 1994; 1:330-9.

3. Weber JE, Chudnofsky CR, Boczar M, Boyer EW, Wilkerson $\mathrm{MD}$, Hollander JE. Cocaine-associated chest pain: how common is myocardial infarction? Acad Emerg Med. 2000; 7:873-7.

4. Lange RA, Cigarroa RG, Yancy CW, et al. Cocaine-induced coronary artery vasoconstriction. N Engl J Med. 1989; 321: 1557-62.

5. Isner JM, Estes M, Thompson PD, et al. Acute cardiac events temporally related to cocaine abuse. N Engl J Med. 1985; 315:1438-43.

6. Mittleman RE, Wetli CV. Cocaine and sudden "natural" death. J Forens Sci. 1987; 32:11-9.

7. Togna G, Tempesta E, Togna AR, Dolci N, Celco B, Caprino L. Platelet responsiveness and biosynthesis of thromboxane and prostacyclin in response to in vitro cocaine treatment. Haemostasis. 1985; 15:100-7.

8. Rezkalla SH, Mazza JJ, Kloner RA, Tillman V, Chang SH. The effects of cocaine on human platelets in health subjects. Am J Cardiol. 1993; 72:243-6.

9. Dressler FA, Malekzadeh S, Roberts WC. Quantitative analysis of amounts of coronary arterial narrowing in cocaine addicts. Am J Cardiol. 1990; 65:303-8.

10. Tardiff K, Gross E, Wu J, Stajic M, Millman R. Analysis of cocaine-positive fatalities. J Forens Sci. 1989; 34:53-63.

11. Hollander JE, Hoffman RS, Burstein JL, Shih RD, Thode HC Jr. Cocaine associated myocardial infarction: mortality and complications. Arch Intern Med. 1995; 155:1081-6.

12. Stenberg RG, Winniford MD, Hillis LD, Dowling GP, Buja LM. Simultaneous acute thrombosis of two major coronary arteries following intravenous cocaine use. Arch Pathol Lab Med. 1989; 113:521-4.

13. Zimmerman FH, Gustafson GM, Kemp HG. Recurrent myocardial infarction associated with cocaine abuse in a young man with normal coronary arteries: evidence for coronary artery spasm culminating in thrombus. J Am Coll Cardiol. 1987; 9:964-8.

14. Moliterno DJ, Lange RA, Gerard RD, Willard JE, Lackner C, Hillis LD. Influence of intranasal cocaine on plasma constituents associated with endogenous thrombosis and thrombolysis. Am J Med. 1994; 96:492-6.

15. Siegel AJ, Sholar MB, Mendelson JH, et al. Cocaine-induced erythrocytosis and increase in vonWillebrand factor: evidence for drug-related blood doping and prothrombotic effects. Arch Intern Med. 1999; 159:1925-30.

16. Sakwa MN, Young AJ, Muza SR, Gonzalez RR, Pandolf KD. Erythrocyte reinfusion and maximal aerobic power. JAMA. 1987; 257:1496-9.

17. Kaufman MJ, Siegel AJ, Mendelson JH, et al. Cocaine administration induces splenic constriction and altered hematologic parameters. J Appl Physiol. 1998; 85:1877-83.

18. Gibler WB, Runyon JP, Levy RC, et al. A rapid diagnostic and treatment center for patients with chest pain in the emergency department. Ann Emerg Med. 1995; 25:1-8.

19. Domenick MA, Ildstad ST. Impact of bone marrow transplantation on type I diabetes. World J Surg. 2001; 10:1007-18.

20. Rabinovitch A, Skyler JS. Prevention of type I diabetes. Med Clin North Am. 1998; 82:739-55.

21. Siegel AJ, Mendelson JH, Sholar MB, et al. Effect of cocaine usage on C-reactive protein, vonWillebrand factor, and fibrinogen. Am J Cardiol. 2002; 89:1133-5.

22. Tavassoli M, Crosby WH. Fate of the nucleus of the erythroblast. Science. 1973; 179:912-3. 